Denn es folgte ein Ruf nach Osnabrück, und dort wurde die Zusendung meiner Bewerbungsmappe anders als in Thüringen mit der Frage beantwortet, wann ich denn anfangen könne. Damit stand auch für meinen Mann fest, welchen Ruf er annahm. Diesen Zusammenhang scheinen Universitäten inzwischen eher zu erkennen als vor zehn Jahren.

So begann ich im Herbst 2000 in der Staatsanwaltschaft Osnabrück als erste Staatsanwältin in Teilzeit zusammen mit einer Kollegin, die fünf Kinder hat. Wir wurden vor allem von der damaligen Behördenleitung eher misstrauisch aufgenommen, gemeinsam in ein Büro gesetzt und teilten uns fortan ein Dezernat. Das war ein Glücksfall, weil wir uns - bis heute - sehr gut verstehen und uns in dieser Zeit gegenseitig nicht nur vertreten, sondern auch unterstützen konnten. Beispielsweise wurde uns nach kurzer Zeit bewusst, dass wir da Teilzeitkräfte - als einzige nicht für den Bereitschaftsdienst eingesetzt wurden und dies den Unmut einiger Kollegen erregte. Daraufhin gingen wir zur Verwaltung und erklärten, wir wollten auch Bereitschaftsdienst machen. Heute arbeiten in der Staatsanwaltschaft Osnabrück viele Staatsanwältinnen - damals gab es nur zwei planmäßige weibliche Kräfte - und eine Reihe von Teilzeitkräften.

2002 wurden wir - weiterhin im Doppelpack - erst für kurze Zeit an das Landgericht und dann an das Amtsgericht Osnabrück versetzt und erbten ein vernachlässigtes Strafund Bußgelddezernat mit unglaublichen Beständen. Mein Protokollführer hat mir damals erklärt, für meinen Verhandlungsstoff von einem Tag würde das Schöffengericht drei Tage brauchen. Von wirklicher Teilzeit konnte keine Rede sein. Jedenfalls habe ich auf diese Weise straffes Verhandeln gelernt und hoffe, dass ich den menschlichen Schicksalen trotzdem halbwegs gerecht werden konnte. Ein Jahr später wechselte ich an das Landgericht in eine Zivilkammer und erhielt dort 2004 eine Planstelle. Meine Kollegin hat sich für das Amtsgericht entschieden und ist eine besonders engagierte Jugendrichterin geworden.

Im selben Jahr wurde ich vorübergehend beurlaubt, weil mein Mann ein Forschungssemester in Oxford verbringen konnte. Wir packten unser Auto voll und fuhren mit der ganzen Familie für ein halbes Jahr nach England. Es war für uns alle eine große Bereicherung und ich bin dankbar dafür, dass ich diese intellektuell anregende Auszeit nehmen konnte.
Nach meiner Rückkehr aus England wurde ich wieder in einer Zivilkammer eingesetzt und nahm an einer Ausbildung in der gerichtsnahen Mediation teil. Dieses Projekt halte ich für eine sehr gute Ergänzung des Justizangebots und habe oft erleben können, wie zufrieden die Parteien nach einer erfolgreichen Mediation das Gericht verlassen.

An einem Freitag im März 2007 erhielt ich einen Anruf aus Oldenburg mit der Anfrage, ob ich im Mai zur sogenannten „Erprobung“ an das Oberlandesgericht kommen wolle, mit Bedenkzeit bis Montag. Das Votum meines Mannes war klar: auf jeden Fall! und so sagte ich unter der Bedingung zu, dass ich nur an drei Tagen anwesend sein müsse. Immerhin ist Oldenburg $100 \mathrm{~km}$ und eineinhalb Zugstunden von Osnabrück entfernt. Das wurde mir auch zugesichert, obwohl in Oldenburg die tägliche Anwesenheit der Richter üblich ist. Wir sorgten dafür, dass montags und dienstags - Vorlesungstage für meinen Mann und Reisetage für mich - eine Haushaltshilfe anwesend war. Alles ging gut, nur einmal wurde mein Mann aus der laufenden Vorlesung geholt, weil sich unser jüngerer Sohn in der Schule den Fuß verstaucht hatte und abgeholt werden musste.

Als ich nach der Erprobung gefragt wurde, ob ich in Oldenburg eine Planstelle haben wolle, sagte ich trotz einiger Bedenken zu, wieder unter der Bedingung, nur an drei Tagen anwesend sein zu müssen. Gleichzeitig stockte ich auf 75 Prozent auf. Die Tätigkeit am Oberlandesgericht hat mir von Anfang an sehr viel Freude gemacht. Sowohl das vertiefte Arbeiten als auch der Austausch mit den Senatskollegen kommt meinen Neigungen entgegen. Ich bin war von Februar 2008 an in einem Familien- und Zivilsenat tätig und konnte auf diese Weise die vielfältigen Reformen im Familienrecht mit Interesse begleiten. Da der Vorsitz in meinem Senat vakant war, leitete ich den Senat acht Monate lang als ständige Vertreterin. Auch das war eine neue Herausforderung.

Im Mai 2010 bin ich zur Richterin am Bundesgerichtshof gewählt worden. Erneut haben wir familiär sehr großes Glück gehabt, weil mein Mann zeitgleich Rufe nach Speyer und Freiburg erhielt. Inzwischen hat er den Ruf nach Freiburg angenommen. Eine solche Doppelkarriere ist weder plan- noch vorhersehbar und wir sind froh und glücklich über die gemeinsame neue Perspektive.

\title{
Hinterher weiß man immer mehr - oder: auch Umwege führen zum Ziel
}

\author{
Sabine Kräuter-Stockton \\ Vorsitzende des Landesverbands Saarland im djb, Mitglied der \\ Kommission Gewalt gegen Frauen und Kinder des djb, Saarbrü- \\ cken; Staatsanwältin (Gruppenleiterin), Staatsanwaltschaft \\ Saarbrücken
}

Dass ausgerechnet ich einmal Staatsanwältin werden und mir diese Arbeit über viele Jahre hinweg große Freude machen würde, damit hätte ich früher am allerwenigsten gerechnet.

Als Abiturientin hatte ich noch überlegt, einen sozialen Beruf zu ergreifen und absolvierte zunächst erst einmal ein 
freiwilliges soziales Jahr. Die Erfahrungen dieses sowohl schönen als auch harten Jahres brachten mich aber von dem ursprünglich anvisierten Berufsweg wieder ab, worauf ich zuerst einmal ein Sprachenstudium am Dolmetscherinstitut der Saarbrücker Uni aufnahm. Fremdsprachen hatten mir schon immer Spaß gemacht und waren mir leicht gefallen, Jura dagegen kam für mich überhaupt nicht in Frage, denn als Tochter eines Rechtsanwalts wollte ich etwas „ganz anderes“ machen. Die heutigen Studentinnen werden die goldenen Zeiten, in denen wir Studierenden der Achtzigerjahre einen Großteil des Tages Kaffee trinkend und philosophierend im Ausländer-Café verbrachten (man musste ja auch noch für Sprach-Praxis sorgen!), vom Hörensagen kennen. Die lockere Struktur des Studiums ermöglichte es zudem, neben dem Studium auch noch den Lebensunterhalt zu verdienen, was nicht nur den Erwerb von Selbstbewusstsein, sondern auch von Lebenserfahrung förderte.

Als ich nach zwei Semestern aus Neugierde mit Bekannten in ein paar juristische Vorlesungen mitgegangen war, schrieb ich mich schließlich doch zusätzlich in Jura ein. Mittlerweile war die Frage der Abgrenzung von den Eltern nicht mehr ausschlaggebend für die Studienwahl. Nach mehreren Semestern Sprachen- plus Jurastudium stellte ich aber - aus heutiger Sicht erwartungsgemäß - fest, dass ich bei diesem Doppelstudium wahrscheinlich in keinem der beiden Fächer je zu einem Abschluss kommen würde. Ich blieb daher zwar weiter in Jura immatrikuliert, konzentrierte mich aber auf das Sprachenstudium und schloss als „Staatlich geprüfte Übersetzerin“ ab. Danach kniete ich mich wieder in das Jurastudium und hatte gerade alle meine Scheine erworben, als ich mit meinem ersten Kind schwanger wurde.

Ich stürzte mich nunmehr mit ebensolchem wissenschaftlichem Eifer wie zuvor auf den „Medicus“ und die „JuS“ auf Kindererziehungs-, Kinderkrankheits- und Kinderernährungsliteratur und die juristischen Bücher verschwanden wieder einmal (vorübergehend) in den oberen Fächern des Wohnzimmerregals. Als mein Sohn mit zwei Jahren einen Platz im UniKindergarten bekam, war ich wie ausgehungert nach komplizierten juristischen Problemen, meldete mich bei einem Repetitor an und begann, fürs Examen zu lernen. Ich brachte fortan meinen Sohn morgens zum Uni-Kindergarten, ging von dort aus sofort auf meinen Stammplatz in der Uni-Bibliothek oder zum Repetitorium und vertiefte mich bis Mittag in das Lernen. Der Rest des Tages gehörte dann der Familie und abends, wenn mein Sohn schlief, war wieder Zeit, um etwas zu wiederholen. Dieser enge zeitliche Rahmen mit einem Zwang zur Konzentration und zum Minimalismus waren für mich genau richtig: Rückblickend habe ich den Eindruck, dass manche Kommilitonen schlechtere Examensnoten erzielten, obwohl sie in der Vorbereitungszeit wesentlich mehr gelernt hatten als ich, weil sie sich in den Klausuren in Spezialproblemen verzettelten, von denen ich noch überhaupt nie gehört hatte.

Mein zweites Kind bekam ich während des Referendarsdienstes, der sich unproblematisch mit Erziehungsurlaub unterbrechen ließ. Während der Vorbereitung auf das zweite
Staatsexamen war dann mein Sohn in der Schule, die kleine Tochter in der Kinderkrippe.

Anfang der Neunzigerjahre wurde ich zu meiner großen Freude in die saarländische Justiz eingestellt und, zu meiner noch größeren Überraschung, erst einmal bei der Staatsanwaltschaft eingesetzt. Überrascht war ich, weil ich während der Ausbildung wenig Freude am Strafrecht entwickelt hatte, und folglich meine Noten im Zivilrecht und Öffentlichen Recht immer deutlich besser gewesen waren. Auch während der Referendarszeit hatte mir die Station bei einem Staatsanwalt, mit dem ich mich menschlich und fachlich nicht gut verstand, keinen Spaß gemacht. Die praktische Arbeit als Staatsanwältin begeisterte mich jedoch schnell: oft spannende, emotional anrührende und aufwühlende Fälle mitten aus dem „prallen Leben“, die Entscheidungsmöglichkeiten, die abwechslungsreiche und anspruchsvolle Tätigkeit zwischen Aktenbearbeitung, eigenen Ermittlungen, Sitzungsdienst vor Gericht - da hatte ich schnell das Gefühl, an der richtigen Stelle zu sitzen. Dennoch nagte als Assessorin an mir der Gedanke, es könnte woanders ja womöglich noch schöner sein. Hatte ich nicht doch als Jurastudentin mit dem Gedanken geliebäugelt, (Zivil-)Richterin zu werden? Daher begrüßte ich es, auch wenn mir der Abschied von der Staatsanwaltschaft schwer fiel, dass ich während der Assessorenzeit ans Landgericht versetzt wurde. Die Arbeit als Richterin in einer Landgerichtskammer war allerdings, nach zwei Jahren der selbstständigen Entscheidungen als Staatsanwältin, doch nicht der Traumberuf für mich. Ich vermisste die Ermittlungstätigkeit, den Trubel, das klingelnde Telefon, die dringenden, schnellen Entscheidungen. Als meine Lebenszeiternennung anstand, bat ich darum, als Staatsanwältin ernannt zu werden.

Nach mehreren Jahren staatsanwaltlicher Tätigkeit in verschiedenen Abteilungen wurde in unserem Justizministerium eine neue Stelle, welche die Bekämpfung häuslicher Gewalt koordinieren und organisieren sollte, eingerichtet, die neben einer Soziologin mit einer Juristin aus der Justiz zu besetzen war. Als mir diese Stelle angetragen wurde, nahm ich gerne an, wobei ich mir - als begeisterte Staatsanwältin - eine Befristung der Abordnung auf zwei Jahre ausbedingen konnte. Die ministerielle Arbeit war nun etwas völlig Neues, ganz anders als das, was ich von der Justiz her gewöhnt war. Es wurde geplant, verworfen, getüftelt, formuliert, taktisch vorausgedacht. Es mussten alle Weiterungen und möglichen Auswirkungen bedacht werden, es musste verhindert werden, dass jemandem aus dem eigenen oder einem fremden Ressort „auf die Füße getreten “ wurde. Ich lernte Vorträge zu halten, wirkte an Schulungen mit, machte Dienstreisen, saß in Arbeitskreisen, moderierte schwierige Gruppen. Die Projekte, die angegangen wurden, realisierten sich manchmal erst nach geraumer Zeit, manchmal nie, könnten eventuell aber je nach Situation wieder aus der Schublade hervorgeholt und doch noch verwertet werden. In der Zeit im Justizministerium lernte ich - obwohl ich schon über ein Jahrzehnt Berufserfahrung hatte - eine ganze Menge Neues, hatte Erfolge und Freude, aber dennoch fehlten mir die unmittelbaren Auswirkungen meiner Arbeit 
auf konkrete Fälle, der Einzelfallbezug. Mir fehlte kriminaltaktisches Vorgehen, Ermittlung des Täters und Entscheidung, ob die Beweislage für eine Anklage ausreicht, das (hoffentlich) faire Streiten vor Gericht, um einen möglichst gerechten Schuldausgleich zu erwirken, der dem Opfer - zumindest zu einem gewissen Maß - Genugtuung verschafft. Nach etwa zweieinhalb Jahren Tätigkeit beim Ministerium in Sachen „Bekämpfung häuslicher Gewalt“ wurde bei der Staatsanwaltschaft durch Pensionierung das Sonderdezernat „häusliche Gewalt“ frei. Diese Gelegenheit konnte ich mir nicht entgehen lassen und kehrte zu „meiner“ Behörde zurück. Das Dezernat „häusliche Gewalt“ platzte mittlerweile - unter anderem als Ergebnis meiner eigenen Öffentlichkeits-, Fortbildungs- und Aufklärungsarbeit im Ministerium - aus allen Nähten, so dass ich in den ersten drei Jahren von der Arbeitsmasse fast erschlagen wurde. Die Gelegenheit, die hohen Standards für die staatsanwaltschaftliche Arbeit in Fällen häuslicher Gewalt, die ich im Ministerium mitentwickelt hatte, selbst in der Praxis umzusetzen, war aber so faszinierend, dass ich mich willig und (zumindest meist) klaglos auch mit Spätschichten und Wochenendarbeit in die Arbeit kniete. Mittlerweile bearbeiten drei Dezernent(inn)en Fälle häuslicher und sexualisierter Gewalt in unserer Behörde, ich wurde zwischenzeitlich zur Gruppenleiterin ernannt, und das Schönste: die Arbeit macht immer noch - jedenfalls meistens - Spaß.
Heute kann ich auf einen überhaupt nicht gradlinigen und zielstrebigen „Karriereweg“ zurückblicken, der mich aber mit einigen Ausreißern in die eine und die andere Richtung genau dahin geführt hat, wo ich nun das machen kann, was mir Freude macht, was mir Befriedigung verschafft und was ich gut kann. Die verschiedenen „Schlenker“ auf andere Gebiete sehe ich heute nicht als Zeitverschwendung, sondern im Gegenteil als wertvolle Gelegenheiten zum Erwerb von Schlüsselkompetenzen, die mir kein Jurastudium, kein Referendariat beigebracht hätten. Das Hineinschnuppern in soziale Tätigkeiten, die Beschäftigung mit fremden Sprachen und Kulturen, nicht zuletzt der (damals in der Vor-Computer-Ära noch sogenannte) „Schreibmaschinen“schein, den ich für den Abschluss am Dolmetscherinstitut benötigte, die Erziehung von zwei Kindern und das Jonglieren zwischen Verpflichtungen der Arbeit einerseits und der Familie andererseits haben mir Kompetenzen verschafft, von denen ich heute bei meiner Arbeit zehre und die auch vom Arbeitgeber anerkannt werden, was sich in der Ernennung als Gruppenleiterin zeigte.

Daher möchte ich alle jungen Kolleginnen motivieren, den Blick über den Tellerrand zu wagen, die Zeit, die dies kostet, als sinnvolle Investition zu verstehen und daran zu denken, dass es sich bei juristischen Sachverhalten um Lebenssachverhalte handelt, die korrekt zu verstehen der Mensch auch Lebenserfahrung, Offenheit und Vorurteilsfreiheit braucht.

\section{Rechtsanwältin und Notarin}

\section{Jutta Wagner}

Präsidentin des djb; Rechtsanwältin und Notarin, Berlin

Der Gedanke, Anwältin zu werden, war zwar während meiner Schulzeit einmal aufgetaucht, obwohl es in der Familie und im näheren Umfeld keine Vorbilder gab. Letztlich jedoch habe ich, wie so viele, 1969 das Jurastudium begonnen, nicht um einen bestimmten juristischen Beruf auszuüben, sondern um zwar auch diese Option zu haben, aber mindestens ebenso, um so oder so nützliche Kenntnisse auch für andere mögliche Laufbahnen zu erwerben.

Über mein Studium kann ich so gut wie nichts berichten, das in irgendeiner Art Vorbildcharakter haben und nachahmenswert sein könnte. Meine Studienjahre, zuerst in Hamburg, dann in Berlin, waren geprägt durch das Aufbegehren gegen überkommene, verkrustete Strukturen. Das hochschulpolitische Engagement und das allgemeinpolitische standen dabei nebeneinander.

Ich wurde zum studentischen Mitglied in einem Selbstverwaltungsgremium gewählt, in dem ich zum Beispiel gemeinsam mit Jutta Limbach an Konzepten zur Studienreform arbeitete. Gemeinsam mit zuerst nur drei anderen Kommilitonen baute ich am Fachbereich Rechtswissenschaft eine sehr schnell wachsende und erfolgreiche Studentengruppe, poli- tisch irgendwo zwischen SPD und Maoisten angesiedelt, auf. Die Organisation von großen Veranstaltungen, das Reden vor Hunderten von Menschen hat mich sicher etwas für das Leben, auch für das Anwältinnenleben, gelehrt, aber zum Erwerb von Rechtskenntnissen doch wenig beigetragen. Das gleiche gilt für die Demonstrationen und Veranstaltungen gegen den Vietnamkrieg.

Hinzu kam, dass ich mir meinen Lebensunterhalt durch fast durchgängige Teilzeitarbeit während des Studiums selbst verdienen musste.

Die Zeit fürs Studieren war also mehr als knapp und ich muss hier gestehen, dass ich an keiner einzigen Lehrveranstaltung kontinuierlich teilgenommen habe, außer an einem rechtshistorischen Seminar später nach dem Ersten Staatsexamen. Ich habe die notwendigen Klausuren und Hausarbeiten geschrieben, um möglichst schnell die vorgeschriebenen Scheine zu erwerben und mich in einer kleinen Arbeitsgruppe dann mit den Skripten von Alpmann-Schmidt auf das Erste Staatsexamen vorbereitet. Dazu habe ich mich dann nach der vorgeschriebenen Mindeststudienzeit, also nach sieben Semestern, angemeldet und es, wie sich leicht vorstellen lässt, mit mäßigem Erfolg bestanden. Aus heutiger Sicht ist es mir ein Rätsel, wie mir das überhaupt gelingen konnte und ich bin der festen Überzeugung, dass solches heute auch nicht mehr möglich wäre. 